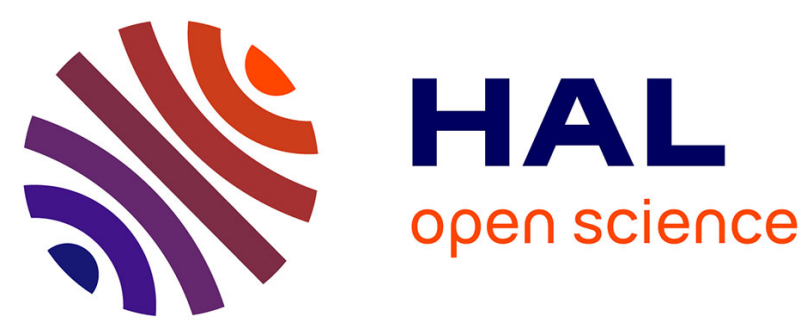

\title{
Autonomous micro-magnet based systems for highly efficient magnetic separation
}

Luiz-Fernando Zanini, Nora Dempsey, Dominique Givord, Gilbert Reyne, Frédéric Dumas-Bouchiat

\section{- To cite this version:}

Luiz-Fernando Zanini, Nora Dempsey, Dominique Givord, Gilbert Reyne, Frédéric Dumas-Bouchiat. Autonomous micro-magnet based systems for highly efficient magnetic separation. Applied Physics Letters, 2011, 99 (23), pp.232504. 10.1063/1.3664092 . hal-00649978

\section{HAL Id: hal-00649978 https://hal.science/hal-00649978}

Submitted on 26 May 2021

HAL is a multi-disciplinary open access archive for the deposit and dissemination of scientific research documents, whether they are published or not. The documents may come from teaching and research institutions in France or abroad, or from public or private research centers.
L'archive ouverte pluridisciplinaire HAL, est destinée au dépôt et à la diffusion de documents scientifiques de niveau recherche, publiés ou non, émanant des établissements d'enseignement et de recherche français ou étrangers, des laboratoires publics ou privés. 


\section{Autonomous micro-magnet based systems for highly efficient magnetic separation}

Cite as: Appl. Phys. Lett. 99, 232504 (2011); https://doi.org/10.1063/1.3664092

Submitted: 27 September 2011 . Accepted: 04 November 2011 . Published Online: 08 December 2011

L. F. Zanini, N. M. Dempsey, D. Givord, G. Reyne, and F. Dumas-Bouchiat

\section{ARTICLES YOU MAY BE INTERESTED IN}

Micromagnet structures for magnetic positioning and alignment

Journal of Applied Physics 111, 07B312 (2012); https://doi.org/10.1063/1.3675067

Thermomagnetically patterned micromagnets

Applied Physics Letters 96, 102511 (2010); https://doi.org/10.1063/1.3341190

Micro-magnetic imprinting of high field gradient magnetic flux sources

Applied Physics Letters 104, 262401 (2014); https://doi.org/10.1063/1.4886375

Challenge us.

What are your needs for periodic signal detection?

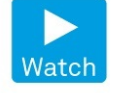

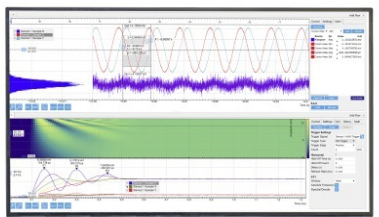

0
Zurich

Instruments 


\title{
Autonomous micro-magnet based systems for highly efficient magnetic separation
}

\author{
L. F. Zanini, ${ }^{1,2, a)}$ N. M. Dempsey, ${ }^{1}$ D. Givord, ${ }^{1}$ G. Reyne,${ }^{2}$ and F. Dumas-Bouchiat ${ }^{1}$ \\ ${ }^{1}$ Institut Néel, CNRS and Université Joseph Fourier, BP 166, F-38042 Grenoble Cedex 9, France \\ ${ }^{2}$ G2ELab, Grenoble Université, BP 46, 38402 St. Martin d'Hères, France
}

(Received 27 September 2011; accepted 4 November 2011; published online 8 December 2011)

\begin{abstract}
The various forces experienced by magnetic particles pumped through microfluidic channels placed above a chessboard array of micromagnets were calculated as a function of particle size and device dimensions. A device incorporating magnetically microstructured hard magnetic $\mathrm{NdFeB}$ films was fabricated. Good agreement was achieved between the calculated and observed distance over which magnetic particles travel before they are trapped. Using this simple and autonomous device, mixed solutions of magnetic and non-magnetic micro-particles were separated into two distinct solutions containing a concentration of up to $99.9 \%$ and $94.5 \%$ of non-magnetic and magnetic particles, respectively. (C) 2011 American Institute of Physics. [doi:10.1063/1.3664092]
\end{abstract}

The range of applications for magnetic micro- and nanoparticles is constantly expanding, in particular in medicine and biology. A number of applications involve particle trapping and deviation, achieved by attracting them to specific locations under the effect of a magnetic field gradient. The required magnetic fields and associated field gradients have been produced using soft magnetic elements polarized by an external magnetic field, ${ }^{1-3}$ electromagnets, ${ }^{4-6}$ or bulk permanent magnets. ${ }^{7,8}$ The field gradients produced by a permanent magnet can be greatly enhanced by reducing its size to the micro-scale, and gradients as high as $10^{6} \mathrm{~T} / \mathrm{m}$ have been recently produced by micro-scale arrays of rare-earth permanent magnets. ${ }^{9}$ Such micro-magnet arrays are autonomous, having no requirements for a cumbersome external field source nor power supply, and have been used to attract liposomes containing magnetic nanoparticles ${ }^{10}$ and to levitate cells. ${ }^{11}$ In this work, we exploit micro-magnet arrays to separate magnetic and non-magnetic particles flowing through a microfluidic channel.

Magnetic separation exploits the difference in the balance of forces acting, on the one side, on non-magnetic objects submitted to gravity (including buoyancy) and viscous forces (neglecting Brownian motion) and on the other side, on magnetic objects (e.g., magnetic particles), additionally submitted to a magnetic force (Fig. 1(a)). Gravity forces are expressed as $F_{g}=(4 / 3) \pi r_{\text {part }}^{3}\left(\rho_{\text {part }}-\rho_{\text {medium }}\right) g$, where $\boldsymbol{\rho}_{\text {part }}$ is the particle mass density, $\boldsymbol{\rho}_{\text {medium }}$ is the medium mass density, and $\mathbf{g}$ is the gravitational acceleration. For a particle of radius $\mathbf{r}_{\text {part }}$ flowing with velocity $\mathbf{v}_{\text {part }}$ in a medium of viscosity $\boldsymbol{\eta}$ and velocity $\boldsymbol{v}_{\text {fluid }}$, the drag force is given by $F_{d}=6 \pi \eta r_{\text {part }}\left(v_{\text {fluid }}-v_{\text {part }}\right)$. Consider now such a particle which has iron oxide nanoparticles embedded in it. In the presence of a magnetic field $\mathbf{H}$, it is submitted to an additional force, due to the action of the field on all the constituent nanoparticles. On one nanoparticle (i), of radius $\mathbf{r}_{\mathbf{n p}(\mathbf{i})}$ and volume $\mathbf{V}_{\mathbf{n p ( i )}}$, the magnetic force is given by

$$
F_{m}=\mu_{0} V_{n p(i)} M_{(i)} \nabla H,
$$

\footnotetext{
${ }^{\text {a) }}$ Author to whom correspondence should be addressed. Electronic mail: luiz-fernando.zanini@grenoble.cnrs.fr.
}

where $\boldsymbol{\mu}_{\boldsymbol{0}}$ is the vacuum permeability and $\mathbf{M}_{(\mathbf{i})}$ the nanoparticle magnetization given by the Langevin function

$$
M_{(i)}=M_{0} L\left(x_{(i)}\right)=M_{0}\left\{\operatorname{coth}\left(x_{(i)}\right)-1 / x_{(i)}\right\},
$$

with $\mathbf{M}_{\mathbf{0}}$ being the $0 \mathrm{~K}$ magnetization and $x=\mu_{O} V_{n p(i)} M_{O} H /$ $k_{B} T$, where $\mathbf{k}_{\mathbf{B}}$ is Boltzmann's constant and $\mathbf{T}$ the temperature. For the particle as a whole, the average magnetization is obtained by summing up the moments of all (n) nanoparticles, and normalizing with respect to the total magnetic volume $\mathrm{V}_{\text {mag }}$, i.e., $M=\frac{M_{0} \sum_{i=1}^{n} P\left(r_{n p(i)}\right) V_{n p(i)} L\left(x_{(i)}\right)}{V_{\text {mag }}}$, where $V_{\text {mag }}=\sum_{i=1}^{n} P\left(r_{n p(i)}\right) V_{n p(i)}$ and $\mathrm{P}\left(\mathrm{r}_{\mathrm{np}(\mathrm{i})}\right)$ is the probability of a given nanoparticle to have the radius $r_{n p(i)}$. The balance of all forces acting on a particle is thus given by the equation of dynamics

$$
(4 / 3) \pi r_{\text {part }}^{3} \rho_{\text {part }} d v_{\text {part }} / d t=F_{d}+F_{g}+F_{m} .
$$

For the cases considered in this paper, the inertia of the particles can be neglected due to the time scale of inertial events compared to other movements of the particles. Thus, the particles can be considered to move at their limiting velocity given by $v_{x}=F_{m x} / 6 \pi \eta r_{\text {part }}, \quad v_{y}=v_{\text {fluid }}+F_{m y} / 6 \pi \eta r_{\text {part }}$, $v_{z}=\left(F_{g}+F_{m z}\right) / 6 \pi \eta r_{\text {part }}$, where $\mathbf{F}_{\mathbf{m x}}, \mathbf{F}_{\mathbf{m y}}$, and $\mathbf{F}_{\mathbf{m z}}$ are the components of the magnetic force along the $\mathrm{x}, \mathrm{y}$, and $\mathrm{z}$-axis, respectively. The displacement of the particles is thus derived using $d x / d t=v_{x}(t), d y / d t=v_{y}(t)$, and $d z / d t=v_{z}(t)$.
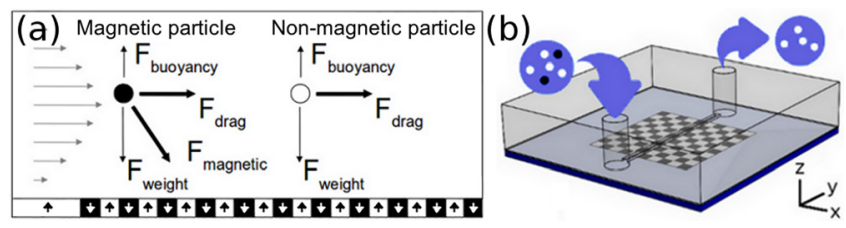

FIG. 1. (Color online) (a) Forces acting on magnetic and non-magnetic particles flowing in a micro-channel integrated above a micromagnet array. (b) Schematic of a micro-magnet/micro-channel device for magnetic separation. 
The magnetic separation device (micro-magnets + microfluidic channel) was modeled and optimized, taking into account the technical constraints concerning micromagnet and microfluidic channel fabrication, for two types of particles, hereafter referred to as "magnetic" since they contain magnetic nanoparticles (Micro Particles $\mathrm{GmbH}, \varnothing$ $2.8 \mu \mathrm{m}$ and Sigma Aldrich, $\varnothing 1.0 \mu \mathrm{m}$ ). These particles may be considered as model elements for future bio-applications, having a size comparable to certain biological entities (e.g. cells, bacteria...). For data analysis, the particle magnetization at $0 \mathrm{~K}$ was taken to be $\mu_{0} \mathrm{M}_{\mathrm{s}}=0.6 \mathrm{~T}$ and in accordance with the manufacturers' specifications, it was assumed that the magnetic nanoparticles account for $30 \%$ of the total particle weight. Fitting of the magnetization curves gave a nanoparticle size of $3.3 \mathrm{~nm} \pm 3 \mathrm{~nm}$ (standard deviation) for the $\varnothing$ $2.8 \mu \mathrm{m}$ particles and $3.6 \mathrm{~nm} \pm 3 \mathrm{~nm}$ for the $\emptyset 1 \mu \mathrm{m}$ particles. Based on these values, an average nanoparticle diameter of $3.5 \mathrm{~nm} \pm 3 \mathrm{~nm}$ was assumed in the calculations. ${ }^{12}$

The micro-magnet arrays considered in the model were based on our experience in micro-magnet fabrication using thermo-magnetic patterning (TMP). ${ }^{13}$ For TMP, we normally use $5 \mu \mathrm{m}$-thick NdFeB films with magnetization $\left(\mu_{0} \mathrm{M}_{\mathrm{r}}=1.2\right.$ $\mathrm{T}$ ) initially oriented out of the film plane (oop). A magnetic field is applied, antiparallel to the orientation of the initial magnetization at the same time that specific regions of the film are heated by a nanosecond pulsed laser beam which irradiates the film through a mask. The magnetization reverses in the irradiated regions over a depth of about 1.3 $\mu \mathrm{m} .{ }^{13}$ In chessboard-like magnetic arrays fabricated by TMP, the lateral size of the individual micro-magnets is typically in the range of $10 \mu \mathrm{m}$ to $1 \mathrm{~mm}$.

In the calculations, we assumed a unique width of 500 $\mu \mathrm{m}$ for the microfluidic channel and considered a maximum capture length of $10 \mathrm{~mm}$. Two possible channel heights were considered, $38 \mu \mathrm{m}$ and $76 \mu \mathrm{m}$, corresponding to, respectively, one and two sheets of a dry-film photoresist commonly used for micro-channel fabrication (DuPont Riston MultiMaster 540). A pre-coating of the magnetic film with a polydimethylsiloxane (PDMS) layer of $6 \mu \mathrm{m}$, to facilitate channel sealing, was considered. See Fig. 1(b) for a schematic of the complete device. Particles entering the channel will be distributed all over the cross-section of the channel and will thus obviously be captured at different positions above the magnets. However, the least-favorable $\mathrm{x}$-axis position for magnetic attraction can be taken as a characteristic parameter of a given device. A trajectory passing through the center of a row of squares, where the magnetic force is weakest, and positioned at the centre of the $\mathrm{x}$-axis of the microfluidic channel, where the fluid velocity is highest, was considered in the calculations.

Initial calculations indicated that magnetic structures with small features (few $\mu \mathrm{m}$ ) and large features (several hundreds of $\mu \mathrm{m}$ ) were the least efficient in trapping particles. Thus, detailed calculations were performed for chessboard structures having feature sizes between $50 \mu \mathrm{m}$ and $200 \mu \mathrm{m}$. Fig. 2(a) shows the simulated trajectories of magnetic particles flowing in $38 \mu \mathrm{m}$-high and $76 \mu \mathrm{m}$-high microfluidic channels, respectively, above three different chessboard magnetic patterns $\left(50 \times 50 \mu \mathrm{m}^{2}, 100 \times 100 \mu \mathrm{m}^{2}\right.$, and $\left.200 \times 200 \mu \mathrm{m}^{2}\right)$. A constant flow rate of $15 \mu \mathrm{l} / \mathrm{min}$ was con-

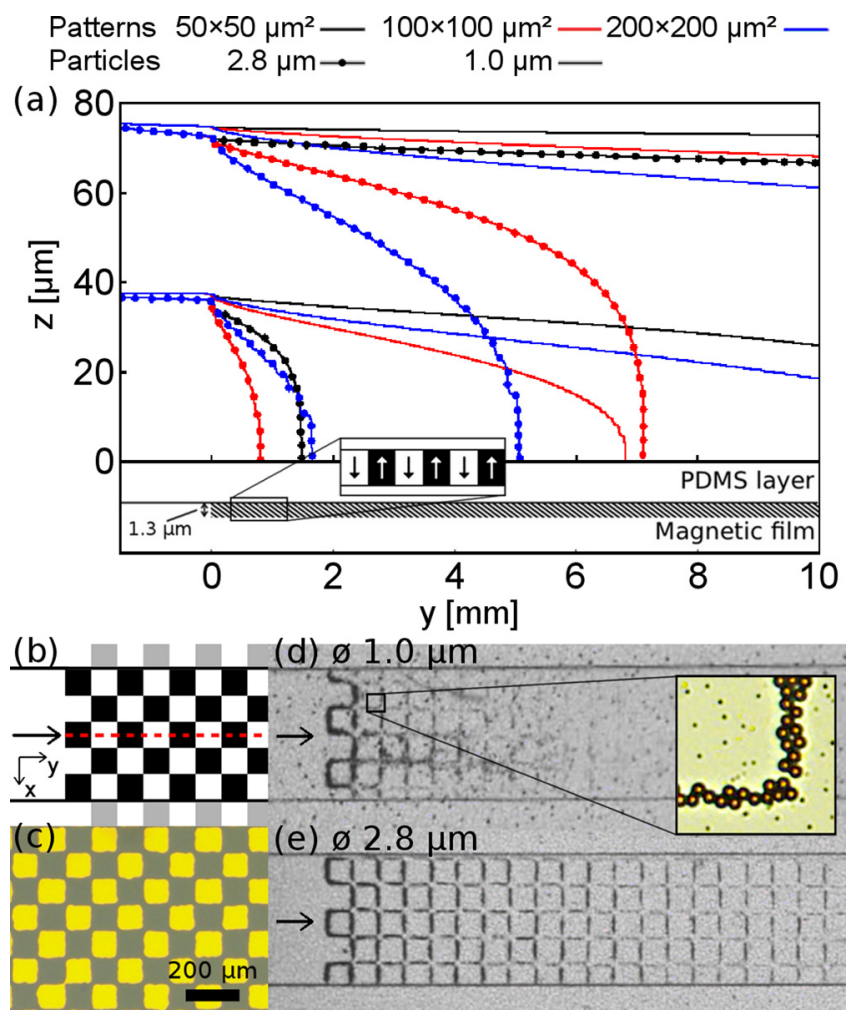

FIG. 2. (Color online) (a) Simulated trajectories of magnetic particles travelling through micro-fluidic channels above arrays of micro-magnets of different size, starting from the top of a $38 \mu \mathrm{m}$ or $76 \mu \mathrm{m}$ thick channel; (b) schematic of the least-favorable lateral position for attraction considered here; (c) Magneto-optical image of an array of $100 \times 100 \mu \mathrm{m}^{2}$ magnets. $^{13}$ $(\mathrm{d}, \mathrm{e})$ Optical images of magnetic particles trapped above such arrays in $38 \mu \mathrm{m}$ thick channels. Inset (d) : zoom on particles attracted to the zones of highest magnetic field gradient. The randomly positioned dark specks observed in (d) are due to cracks in the Ta capping layer which have no adverse effects on the magnets on the time-scale of these experiments.

sidered. The least-favorable position considered in the simulations, as described above, is schematically represented in Fig. 2(b). In the case of $38 \mu \mathrm{m}$-high channels, both types of particles, $(\varnothing 1 \mu \mathrm{m}$ and $\varnothing 2.8 \mu \mathrm{m})$ are predicted to be captured by the $100 \mu \mathrm{m}$ chessboard structure within the considered maximum length of $10 \mathrm{~mm}$. For the same channel height, the $\varnothing 1 \mu \mathrm{m}$ beads are not captured by either the $50 \mu \mathrm{m}$ or by the $200 \mu \mathrm{m}$ structures. For the case of $76 \mu \mathrm{m}$-high channels, the systems with the larger features $(100 \mu \mathrm{m}$ and $200 \mu \mathrm{m})$ are predicted to capture the $\varnothing 2.8 \mu \mathrm{m}$ particles, but not the $\varnothing 1$ $\mu \mathrm{m}$ particles. The system with feature dimensions of $50 \mu \mathrm{m}$ is not suited to capture either of the particles.

The results above show that, among the analyzed configurations, the structures which are the most efficient to capture the considered particles are those having a feature size of $100 \mu \mathrm{m}$. The magnetic separation devices were produced based on these results. Arrays of chessboard-like $100 \times 100$ $\mu \mathrm{m}^{2}$ micro-magnets were produced by thermo-magnetic-patterning over an area of $10 \times 10 \mathrm{~mm}^{2}$ of an out of plane textured $5 \mu \mathrm{m}$ thick $\mathrm{NdFeB}$ film. In the magneto-optic image of the chessboard pattern (Fig. 2(c)), the black and yellow squares represent magnetization pointing out of the film plane and into the film plane, respectively. A $6 \mu$ m-thick layer of PDMS (measured with a DEKTAK 6M profilometer) was spin-coated onto the magnetic film so as to isolate the film from the flowing solution and to facilitate sealing 
of the microfluidic channel onto the film. Parallelepiped microfluidic channels (width $=500 \mu \mathrm{m}$, height $=38 \mu \mathrm{m}$, and length $=15 \mathrm{~mm}$ ) produced by replica molding were then sealed onto the PDMS layer above the micro-magnet array.

Solutions containing the magnetic particles were pumped through the device. The flow rate in the channel was fixed at $15 \mu \mathrm{l} / \mathrm{min}$, determined by means of a pressure controller (Fluigent MAESFLO Control System) connected to the inlet of the channel. Optical images of the trapped particles are shown in Figures 2(d) $(\varnothing 2.8 \mu \mathrm{m})$ and 2(e) ( $\varnothing 1.0$ $\mu \mathrm{m})$. These were obtained after the solutions were pumped through the channels for $20 \mathrm{~s}$. The particles are trapped at the bottom of the channel at the interfaces between oppositely magnetized sections of the film, where the field gradient and thus the attractive magnetic force is maximum (inset of Fig. 2(d)). The difference in the distance required to trap the particles is reflected in their distribution along the channel length. The $\varnothing 2.8 \mu \mathrm{m}$ particles are trapped at the beginning of the pattern, over a distance of approximately $600 \mu \mathrm{m}$ (the simulations in Fig. 2(a) predicted trapping at a distance of $700 \mu \mathrm{m}$ ). For $\varnothing 1 \mu \mathrm{m}$ particles, trapping occurred over a distance of a few millimeters, in agreement with the above simulations (trapping predicted at $7 \mathrm{~mm}$ ). Unpinning of the particles can be achieved by increasing the flow rate and thus the drag force.

The efficiency of the system for separating magnetic particles from non-magnetic ones was then studied. Two initial solutions were used, solution A0 containing the $\varnothing 2.8 \mu \mathrm{m}$ magnetic particles and $\varnothing 3.0 \mu \mathrm{m}$ non-magnetic particles (Sigma Aldrich) and solution B0 containing the $\varnothing 1.0 \mu \mathrm{m}$ magnetic particles and $\varnothing 2.0 \mu \mathrm{m}$ non-magnetic particles (Sigma Aldrich). In both cases, the magnetic particles counted for approximately $35 \%$ of the total number of particles. These solutions were passed through the channel at a flow rate of $\sim 15 \mu \mathrm{l} / \mathrm{min}$ and the resulting solutions (A1 and B1) of the non-trapped particles were collected at the outlet. Distilled water was then passed through the channel at a very high flow rate $(\sim 1000 \mu \mathrm{l} / \mathrm{min})$ to unpin the trapped particles, which were then collected at the outlet (A2 and B2). Figure 3 shows the flow cytometry analysis of all solutions. The measured initial ratios of magnetic to non-magnetic particles are in agreement with their expected concentrations (A0 and B0) while the high purity of solutions A1 and B1 (99.9\% of non-magnetic particles) confirms that the magnetic particles are very effectively trapped. The solutions of the trapped particles are also relatively pure $(94.7 \%$ and $99.5 \%$ for A2 and $\mathrm{B} 2$, respectively). The presence of non-magnetic particles in these solutions is attributed to a steric effect, as the trapped magnetic particles may block the movement of the non-magnetic particles along the bottom of the channel. This effect is less noticeable for solution B, since the barrier created by the $1 \mu \mathrm{m}$ magnetic particles is smaller.

To summarize, we have modeled and demonstrated the efficiency of micro-fluidic systems incorporating high performance micro-magnets for the selective trapping of magnetic particles which are realistic models for cells functionalized with superparamagnetic nano-particles. The autonomous nature of the micro-magnets, which require neither external magnetic field nor power source, enables the use of such devices in combination with analytical equipment where sample

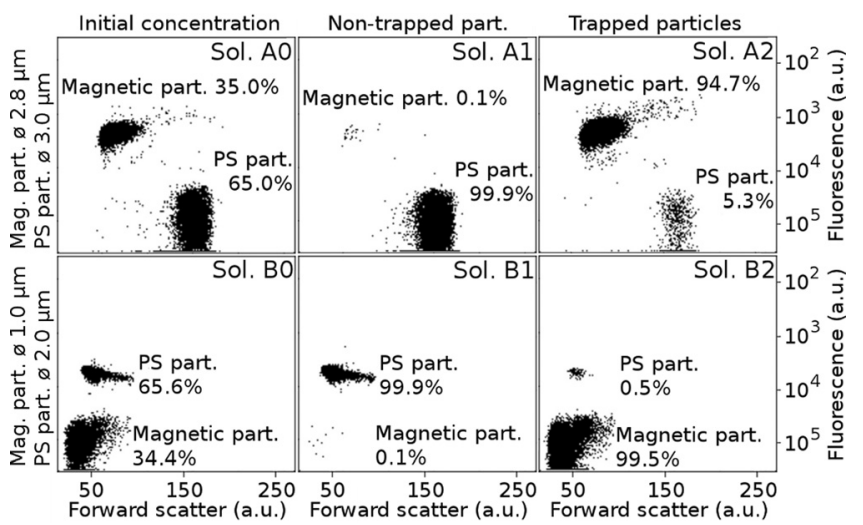

FIG. 3. Cytometric analysis of the starting solutions containing both magnetic and non-magnetic particles (solutions A0, B0-first column), those containing the non-trapped particles (solutions A1, B1-second column) and those containing the trapped particles (solutions A2, B2-third column).

space is restricted (e.g., microscopes, spectrometers...). The stray fields of micro-magnets are essentially restricted to the region of interest, the micro-fluidic channel, thus undesirable stray fields in the environment of the device are negligible. In addition to separating entities as a function of their nature (magnetic vs. non-magnetic) or as a function of their size/ magnetization if magnetic, such devices can be used for the controlled positioning of magnetically functionalized entities. ${ }^{14}$ Beyond lab-on-chip applications in biology, the micromagnet arrays studied here could also be exploited in the fields of chemistry (e.g., migration-based analytical methods) and physics (e.g., atom chips).

The authors acknowledge M. Pezet and C. Villiers from Inst. A. Bonniot (France) for flow cytometry measurements and the financial support from the Agence Nationale de la Recherche (ANR - CESA—013-01, EMERGENT Project).

${ }^{1}$ R. Afshar, Y. Moser, T. Lehnert, and M. A. M. Gijs, Sens. Actuators B 154, 73 (2011).

${ }^{2}$ P. Tseng, D. Di Carlo, and J. W. Judy, Nano Lett. 9, 3053 (2009).

${ }^{3}$ T. Henighan, D. Giglio, A. Chen, G. Vieira, and R. Sooryakumar, App. Phys. Lett. 98, 103505 (2011).

${ }^{4}$ Q. Ramadan, D. P. Poenar, and C. Yu, Microfluid. Nanofluid. 6, 53 (2009).

${ }^{5}$ C. Derec, C. Wilhelm, J. Servais, and J.-C. Bacri, Microfluid. Nanofluid. 8, 123 (2010).

${ }^{6}$ T. Courcier, H. Joisten, P. Sabon, S. Leulmi, T. Dietsch, J. Faure-Vincent, S. Auffret, and B. Dieny, Appl. Phys. Lett. 99, 093107 (2011).

${ }^{7}$ D. Robert, N. Pamme, H. Conjeaud, F. Gazeau, A. Iles, and C. Wilhelm, Lab Chip 11, 1902 (2011).

${ }^{8}$ K. Hoshino, Y.-Y. Huang, N. Lane, M. Huebschman, J. W. Uhr, E. P. Frenkel, and X. Zhang, Lab Chip 11, 3449 (2011).

${ }^{9}$ M. Kustov, P. Laczkowski, D. Hykel, K. Hasselbach, D. Dumas-Bouchiat, D. O'Brien, P. Kauffmann, R. Grechishkin, D. Givord, G. Reyne et al., J. Appl. Phys. 108, 063914 (2010).

${ }^{10}$ J. Pivetal, O. Osman, C. Vezy, M. Frénéa-Robin, F. Dumas-Bouchiat, N. M. Dempsey, D. Givord, P. Simonet, F. Buret, G. Reyne, and N. Haddour, AIP Conf. Proc. 1311, 192 (2010).

${ }^{11}$ P. Kauffmann, A. Ith, D. O'Brien, V. Gaude, F. Boué, S. Combe, F. Bruckert, B. Schaack, N. M. Dempsey, V. Haguet, and G. Reyne, Lab Chip 11, 3153 (2011).

${ }^{12}$ See supplementary material at http://dx.doi.org/10.1063/1.3664092 for the fits of the magnetization curves.

${ }^{13}$ F. Dumas-Bouchiat, L. F. Zanini, M. Kustov, N. M. Dempsey, R. Grechishkin, K. Hasselbach, J. C. Orlianges, C. Champeaux, A. Catherinot, and D. Givord, Appl. Phys. Lett. 96, 102511 (2010).

${ }^{14}$ L. F. Zanini, O. Osman, M. Frenea-Robin, N. Haddour, N. M. Dempsey, G. Reyne, and F. Dumas-Bouchiat, "Micro-magnet structures for magnetic positioning and alignment," J. Appl. Phys. (accepted). 